\title{
HÁBITO ALIMENTAR DE Oligosarcus robustus MENEZES, 1969 E DE Oligosarcus jenynsii (GÜNTHER, 1864), NO SUL DO ESTADO DO RIO GRANDE DO SUL
}

\author{
MARTA RAHAL SILVEIRA ${ }^{1}$, MARLISE DE A. BEMVENUTI ${ }^{2} \&$ ALEX MORESCO ${ }^{3}$ \\ Universidade Federal do Rio Grande - Instituto de Oceanografia, Caixa Postal 474 - Rio Grande, RS, Brasil. 96.201-900, \\ 'martarahal@bol.com.br, ${ }^{2}$ docmab@furg.br, ${ }^{3}$ alexbio@bol.com.br.
}

\begin{abstract}
As espécies de dentudo Oligosarcus robustus e $O$. jenynsii foram analisadas com o objetivo de caracterizar os padrões de diversificação morfológica e as estruturas relacionadas com a alimentação, assim como, determinar o hábito alimentar dos exemplares que ocorrem nas lagoas costeiras no extremo sul do Brasil. Os indivíduos foram capturados em dois períodos (julho e dezembro de 2001), nas lagoas Flores, Nicola, Jacaré, Mangueira norte (banhado do Taim) e Mangueira sul. O espectro alimentar foi determinado através dos métodos de frequência de ocorrência (FO\%) e análise gravimétrica (G\%). Para análise alimentar foram utilizados 164 indivíduos, dos quais 67 apresentaram estômagos vazios. Os itens alimentares mais frequentes na dieta de $O$. robustus foram insetos $\mathrm{FO}=58,6 \%$, peixes $\mathrm{FO}=46,3 \%$ e crustáceos $\mathrm{FO}=12,2 \%$. Na dieta de $O$. jenynsii os itens mais frequentes foram insetos $\mathrm{FO}=83,9 \%$, crustáceos $\mathrm{FO}=35,7 \%$ e peixes $\mathrm{FO}=26,8 \%$. No verão o item insetos apareceu com a maior frequência para as duas espécies, sendo dominantes Ephemeroptera e Odonata. No inverno o item peixes foi frequente na dieta de $O$. robustus, enquanto $O$. jenynsii manteve os mesmos grupos de insetos como item preferencial da dieta. A estratégia alimentar foi determinada através do método gráfico de Amundsen, sendo generalista para $O$. jenynsii, cujo item preferencial foi insetos durante todo o ano e generalista oportunista para $O$. robustus, preferindo insetos no verão e peixes no inverno.
\end{abstract}

RESUMO

PALAVRAS CHAVE: alimentação, morfologia, Characidae, Oligosarcus

ABSTRACT

Feeding habit of Oligosarcus robustus Menezes, 1969 and Oligosarcus jenynsii (Günther, 1864) in the South of Rio Grande do Sul State

Oligosarcus robustus and $O$. jenynsii that inhabit the coastal lagoons of the extreme southern Brazil were characterized according to their morphologic variation and feeding structures as well as to their feeding habit. Specimens were collected in two periods (July and December 2001) in the Flores, Nicola, Jacaré and Mangueira lagoons. The food range was determined using the frequency of occurrence (FO\%) and gravimetric methods (G\%). A total of 164 specimens were analyzed, 67 of which had empty stomachs. The most frequent food items for $O$. robustus were insects $\mathrm{FO}=58,6 \%$, fish $\mathrm{FO}=46,3 \%$ and crustaceans $\mathrm{FO}=12,2 \%$. For $O$. jenynsii insects were dominant $\mathrm{FO}=83,9 \%$, followed by crustaceans $\mathrm{FO}=35,7 \%$ and fish $\mathrm{FO}=26,8 \%$. Insects were more frequent during summer for both species, Ephemeroptera and Odonata were the dominant groups. Fish were the main item in the diet of $O$. robustus during winter while $O$. jenynsii maintained the preference for the same group of insects during the cold season. The feeding strategy determined by the Amundsen graphic method showed that $O$. jenynsii is generalist preferring insects throughout the year while $O$. robustus is generalist opportunistic choosing insects during summer and fish in the wintertime.

KEYWORDS: feeding, morphology, Characidae, Oligosarcus

\section{INTRODUÇÃO}

O gênero Oligosarcus apresenta uma ampla distribuição geográfica sendo encontrado, frequentemente, em arroios, rios e lagoas costeiras do Brasil, Uruguai, Bolívia e Argentina (Menezes 1969, 1987). Duas espécies, Oligosarcus robustus Menezes, 1969 e Oligosarcus jenynsii (Günther, 1864), conhecidos popularmente como "dentudo, branca, peixe cachorro ou tambica" ocorrem no extremo sul do Brasil, principalmente nas lagoas costeiras e na região alagada denominada Banhado do Taim (Burns et al. 2006, Garcia et al. 2006a). Neste local predomina um sistema de banhados de águas calmas e rasas (Motta Marques et al. 2002) que formam, em parte, a Reserva Ecológica do Taim. Esta região integra a parte sul da planície costeira do Rio Grande do Sul, situada entre Torres, RS, Brasil e La Coronilla, Uruguai (Delaney 1965), tendo em sua porção mediana o complexo lagunar Patos-Mirim (Fig. 1). 


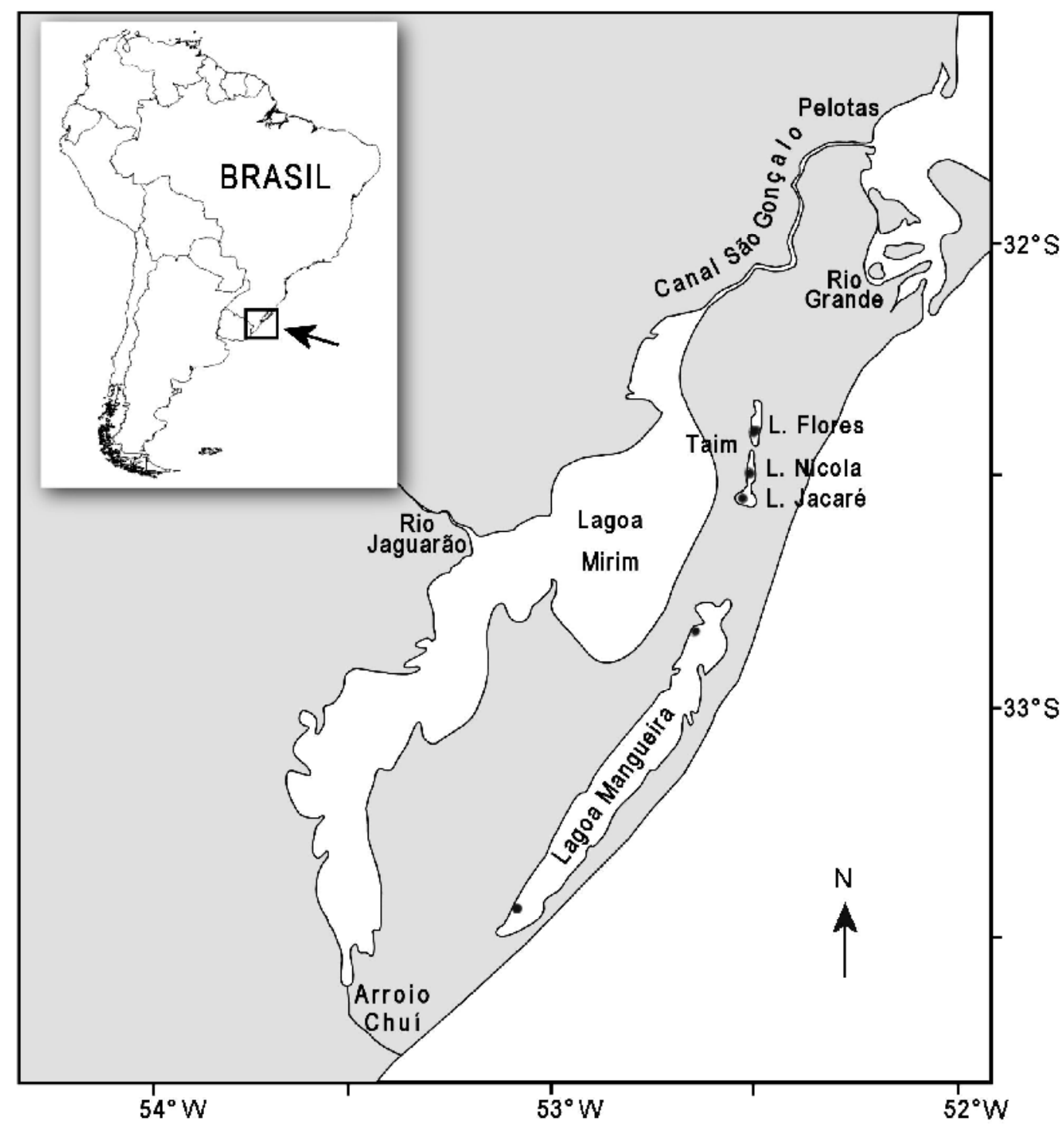

FIGURA 1 - Mapa com indicação das lagoas costeira ao sul do estado do Rio Grande do Sul, Brasil; os pontos pretos indicam os locais de amostragem.

As informações sobre as duas espécies de Oligosarcus, obtidas nas lagoas costeiras ao sul do estado do Rio Grande do Sul, estão relacionadas com dados de descrição sistemática e ocorrência (Burns et al 2006, Garcia et al. 2006 a, b). Registros sobre a ecologia trófica são obtidos para as espécies das lagoas costeiras ao norte do estado do RS (Hartz et al. 1996, Nunez \& Hartz 2006) e do alto rio Uruguai (Hermes-Silva et al. 2004).

Análises da alimentação em peixes, nas lagoas costeiras ao sul do estado do RS, vem sendo desenvolvidos com o propósito de caracterizar e comparar as assembléias entre as diferentes "unidades de habitats" (Burns et al. 2006, Garcia et al. 2006 a, b), através do Programa Ecológico de Longa Duração, PELD - Sistema Hidrológico do Taim (PELD/MCT/MEC/MMA).

Levando em consideração a ausência de informações sobre a alimentação dos dentudos (Oligosarcus robustus e $O$. jenynsii), nas lagoas costeiras ao sul do estado, o presente trabalho tem por objetivo determinar 0 hábito alimentar, caracterizar as estruturas relacionadas com a alimentação e analisar os padrões de diversificação morfológica entre as espécies, a fim de investigar a natureza de sua coexistência neste ambiente. 


\section{MATERIAIS E MÉTODOS}

O trabalho foi desenvolvido nas lagoas

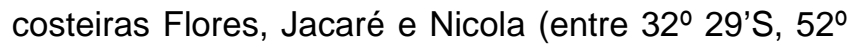
$30^{\prime} \mathrm{W}$ e $\left.32^{\circ} 31^{\prime} \mathrm{S}, 52^{\circ} 31^{\prime} \mathrm{W}\right)$, Mangueira norte $\left(32^{\circ}\right.$

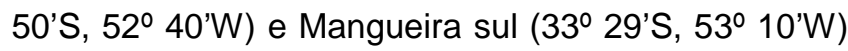
no sul do estado do Rio Grande do Sul (Fig. 1), em dois períodos: (1) 4 e 5 de julho de 2001 e (2) 11 e 12 de dezembro de 2001.

Os exemplares analisados foram coletados com tarrafas e redes de espera tipo painel com malhas de 15, 20, 30, $35 \mathrm{~mm}$ entre nós, colocadas geralmente à tardinha e recolhidas no dia seguinte. $\mathrm{O}$ tempo de permanência das redes de espera na água variou de 17 a 19h (lagoas Nicola, Jacaré, Flores e Mangueira sul) e de 21 a $22 \mathrm{~h}$ (Mangueira norte). Também foi obtida a temperatura da água no momento de retirada da rede.

Foram capturados 164 indivíduos, sendo 760 . robustus (33 em julho/01 e 43 em dezembro/01) e 88 O. jenynsii (44 em julho/01 e 44 em dezembro/01). Parte deste material encontra-se depositado na Coleção de Peixes da FURG (Laboratório de Ictiologia - Instituto de Oceanografia).

Para a análise morfológica foram utilizadas as seguintes medidas em milímetros, comprimento padrão (CP), medido do início do focinho até o final da placa hipural; comprimento da cabeça (medida até o final do opérculo); comprimento do focinho (medido até a borda anterior do olho); largura da boca (medida na maior largura); comprimento do maxilar (medido da borda ântero-superior do pré-maxilar até o final do maxilar de forma inclinada); comprimento da mandíbula (medido da parte anterior do dentário até junto à borda inferior da maxila); diâmetro horizontal do olho e distância interorbital (medida superior entre os dois olhos). Foram contados os rastros branquiais superiores, inferiores e totais, as escamas da linha lateral e os raios da nadadeira anal.

Para a análise alimentar o exemplar foi pesado individualmente, extraído seu estômago e colocado em papel absorvente para retirar o excesso de umidade. Os itens alimentares foram colocados em uma placa de Petri para separação e identificação ao menor nível taxonômico possível, sendo cada grupo pesado separadamente. Os itens alimentares foram analisados através dos métodos de frequência de ocorrência (FO) e gravimétrico (G) (Hyslop 1980). A freqüência de ocorrência $(F O)$ é o número de tratos alimentares $(\mathrm{N})$ que contém um determinado item, expresso em percentagem do número total $(\mathrm{Nt})$ de tratos alimentares cheios. A análise gravimétrica, $(\mathrm{G})$ é o peso total de um item expresso em percentagem do peso médio total $(\mathrm{Pt})$ de todos os itens no trato alimentar.

A análise da estratégia alimentar foi feita com a aplicação do método gráfico de Amundsen, que compara a frequência de ocorrência (FO) com o índice de abundância específica em peso (Pi\%). Este último é definido como a porcentagem do peso relativo de um item alimentar em relação ao peso total de itens alimentares dos estômagos que contém o item (Amundsen et al. 1996). O gráfico resultante indica, por meio da distribuição dos pontos, qual o item alimentar de maior importância na dieta e qual a estratégia alimentar utilizada pela espécie.

\section{RESULTADOS}

A temperatura da água no momento de retirada das redes de espera foi de $10^{\circ} \mathrm{C}$ no ponto Mangueira sul, 11 a $12^{\circ} \mathrm{C}$ na Mangueira norte, Flores, Nicola, Jacaré no período de inverno, enquanto no verão foi $22^{\circ} \mathrm{C}$ na Mangueira sul, 23 a $24^{\circ} \mathrm{C}$ na Mangueira norte, Flores, Nicola, Jacaré.

\section{Análise morfológica}

O tamanho dos exemplares analisados variou entre 100 e 260mm de comprimento padrão (CP) para O. robustus $(n=76)$ e entre 114 e $235 \mathrm{~mm} \mathrm{CP}$ para O. jenynsii $(\mathrm{n}=88)$ (Tabela 1$)$. 
TABELA 1 - Dados morfométricos e merísticos para $O$. robustus e $O$. jenynsii; medidas expressas no comprimento padrão (CP \%); min=valor mínimo, max= valor máximo, med=valor médio, des= desvio padrão, CV= coeficiente de variação.

Oligosarcus robustus Oligosarcus jenynsii

\begin{tabular}{|c|c|c|c|c|c|c|c|c|c|c|c|c|}
\hline & $\mathbf{n}$ & $\min$ & $\max$ & med & des & $\mathrm{CV}$ & $\mathrm{n}$ & $\min$ & $\max$ & med & des & CV \\
\hline $\mathrm{CP}(\mathrm{mm})$ & 76 & 100 & 260 & & & & 88 & 114 & 235 & & & \\
\hline
\end{tabular}

Percentagens do comprimento padrão (\%)

$\begin{array}{lcccccccccccc}\text { Compr. cabeça } & 76 & 19,7 & 32,7 & 27,2 & 1,79 & 6,57 & 88 & 16,5 & 27,8 & 21,1 & 1,39 & 6,58 \\ \text { Compr. focinho } & 76 & 5,8 & 11,1 & 9,2 & 0,98 & 10,67 & 88 & 4,8 & 7,6 & 6,3 & 0,56 & 8,86 \\ \text { Largura boca } & 76 & 5,6 & 8,7 & 7,4 & 0,65 & 8,71 & 88 & 4,7 & 7,7 & 6,2 & 0,62 & 10,03 \\ \text { Compr. maxila } & 76 & 11,6 & 18,9 & 16,8 & 1,35 & 8,03 & 88 & 9 & 16,1 & 12,6 & 0,97 & 7,72 \\ \text { Compr. mandíbula } & 76 & 9,5 & 17,2 & 15,4 & 1,4 & 9,13 & 88 & 8,3 & 15 & 11,7 & 0,91 & 7,76 \\ \text { Diâmetro olho } & 76 & 4,3 & 9,1 & 6,3 & 1,05 & 16,71 & 88 & 3,6 & 7,2 & 5,6 & 0,56 & 10,03 \\ \text { Dist. interorbital } & 76 & 4,7 & 7,9 & 6,5 & 0,72 & 10,95 & 88 & 4,5 & 6,8 & 5,4 & 0,55 & 10,16\end{array}$

\section{Contagens}

\begin{tabular}{lcccccccccccc} 
Brânquias sup & 10 & 8 & 13 & 11 & 1,3 & 12,6 & 10 & 8 & 10 & 9 & 0,8 & 8,5 \\
Brânquias inf & 10 & 12 & 16 & 14 & 1,3 & 9,6 & 10 & 11 & 16 & 14 & 1,5 & 10,4 \\
Rastros totais & 10 & 20 & 28 & 25 & 2,4 & 9,7 & 10 & 19 & 26 & 23 & 2 & 8,8 \\
Esc. L.Lateral & 30 & 75 & 88 & 79 & 3,74 & 4,74 & 30 & 51 & 67 & 56 & 3,23 & 5,83 \\
Raios nad. anal & 30 & 27 & 33 & 30 & 1,33 & 4,56 & 30 & 25 & 29 & 28 & 0,96 & 3,52 \\
\hline
\end{tabular}

Oligosarcus robustus apresenta o perfil superior da cabeça levemente côncavo, passando a convexo na região pré-dorsal, após o processo supraoccipital. O perfil ventral é arredondado (Fig. 2). O focinho longo e a boca grande refletem o maior comprimento da maxila e da mandíbula (média de $16,8 \%$ e $15,4 \%$ do comprimento padrão - CP, respectivamente) (Tabela 1) Possui um par de caninos bem desenvolvidos na ponta do pré-maxilar, seguido por uma fileira de cinco dentes cônicos menores e outro canino desenvolvido como o primeiro em cada lado (Fig. 3). O par de caninos anteriores do pré-maxilar permanecem fora da boca, mesmo quando esta se encontra fechada. A mandíbula também apresenta um par de caninos bem desenvolvidos na frente. A linha lateral é completa com 75 a 88 escamas pequenas; a nadadeira anal apresenta entre 27 e 33 raios; o primeiro arco branquial possui entre oito e 13 rastros superiores, 12 e 16 rastros inferiores, totalizando 20 a 28 rastros totais (Tabela 1). 


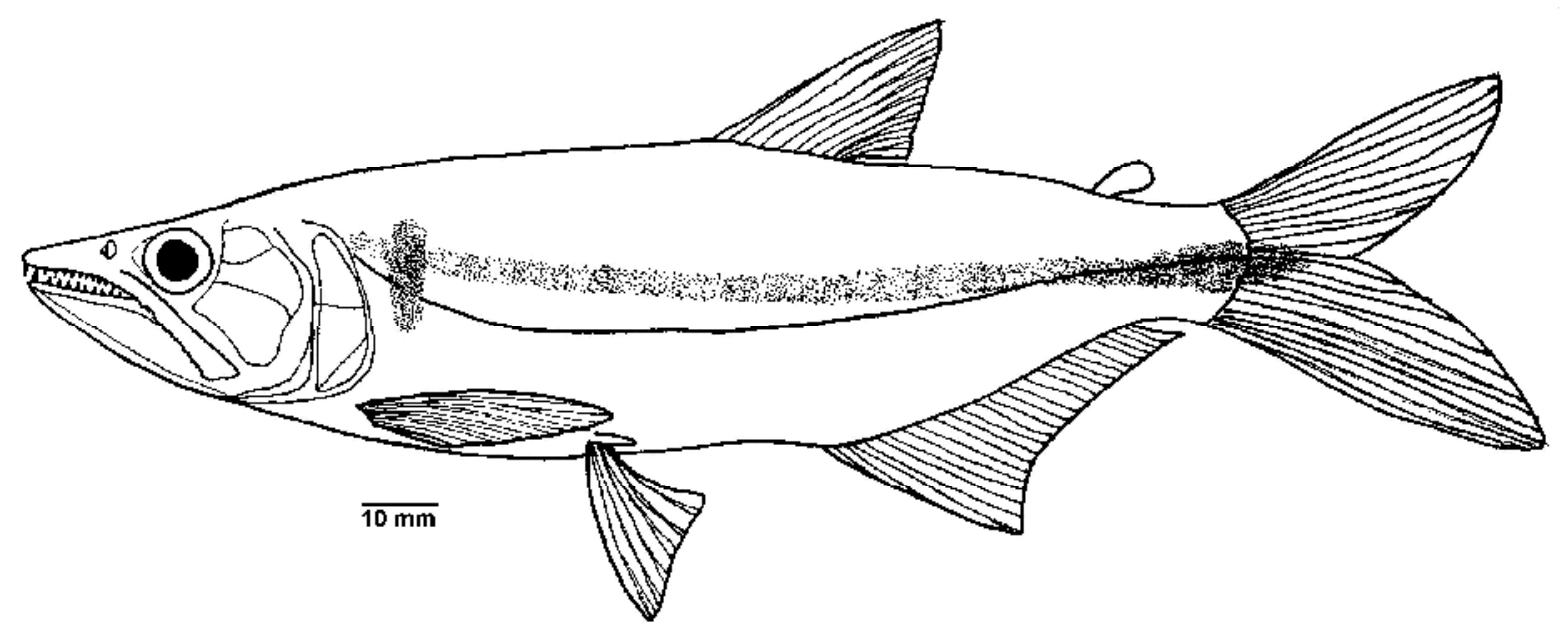

FIGURA 2 - Desenho esquemático indicando a morfologia de Oligosarcus robustus.

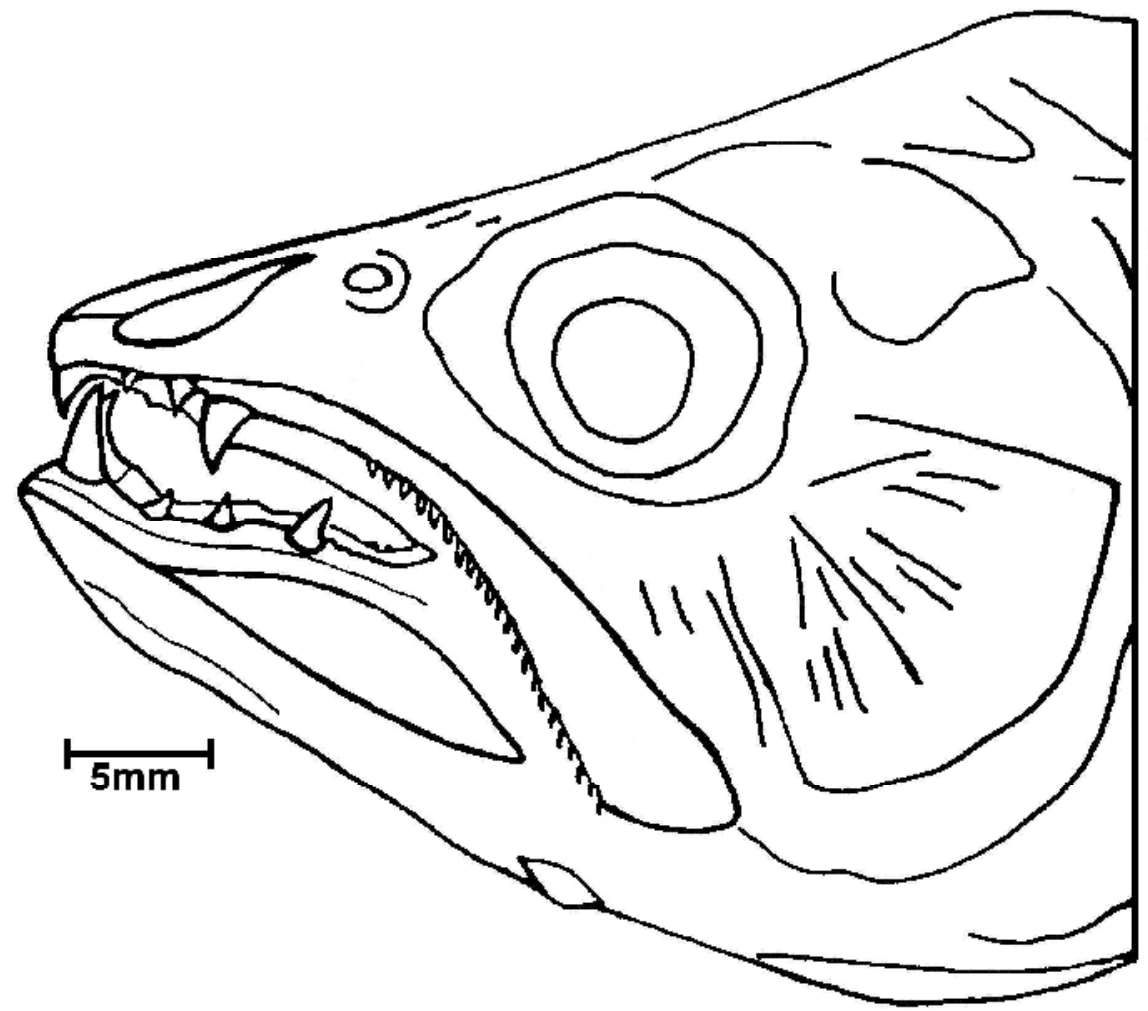

FIGURA 3 - Desenho esquemático da cabeça de Oligosarcus robustus com indicação dos dentes.

Oligosarcus jenynsii apresenta o perfil superior e o ventral da cabeça, aproximadamente simétricos. O perfil superior é reto, tornando-se levemente convexo na região pré-dorsal, após o processo supraoccipital (Fig. 4). O focinho é curto, cabendo mais que três vezes no comprimento da cabeça, resultando em menor comprimento da maxila e mandíbula (respectivamente $12,6 \%$ e $11,7 \% \quad \mathrm{CP}$ ), quando comparado com $O$. robustus (Tabela 1). Possui um par de caninos bem desenvolvidos na ponta do pré- maxilar, seguindo por uma fileira de cinco dentes cônicos menores e outro canino desenvolvido como o primeiro em cada lado (Fig. 5). O par de caninos anteriores do pré-maxilar penetram em uma pequena perfuração presente na parte anterior da mandíbula quando a boca se encontra fechada. A mandíbula também apresenta um par de caninos bem desenvolvidos na frente. A linha lateral é completa, com 51 a 67 escamas grandes; a nadadeira anal apresenta entre 25 e 29 raios; o primeiro arco 
branquial possui entre oito e 10 rastros superiores, 11

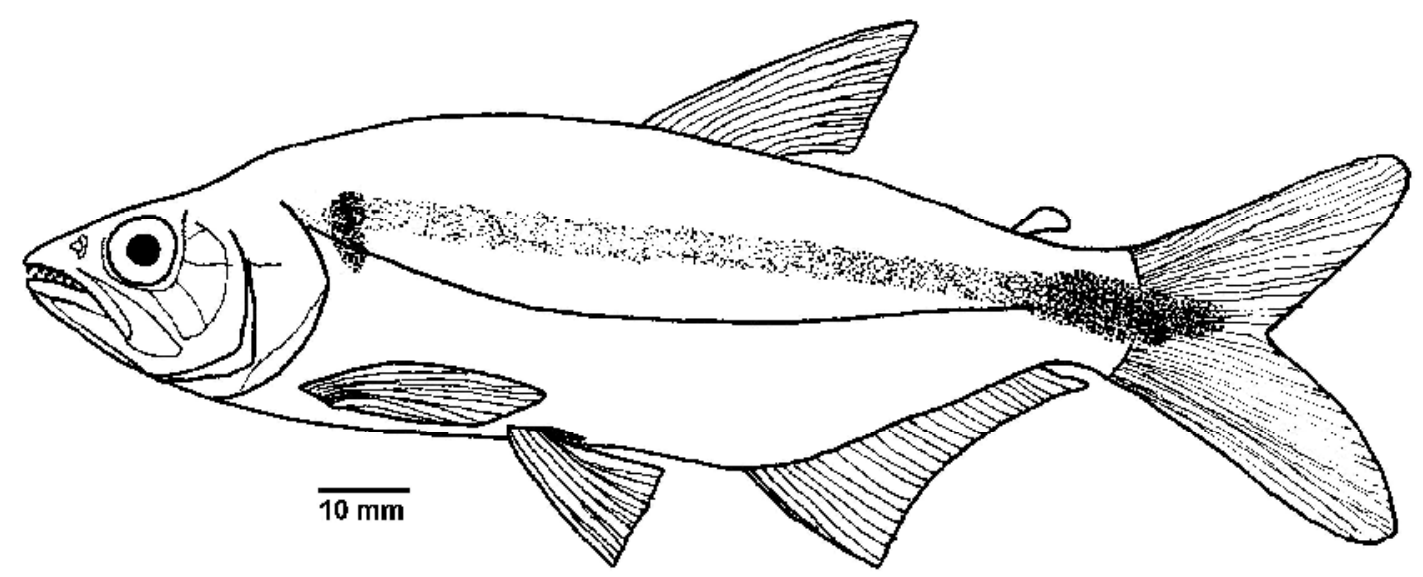

FIGURA 4 - Desenho esquemático indicando a morfologia de Oligosarcus jenynsii.

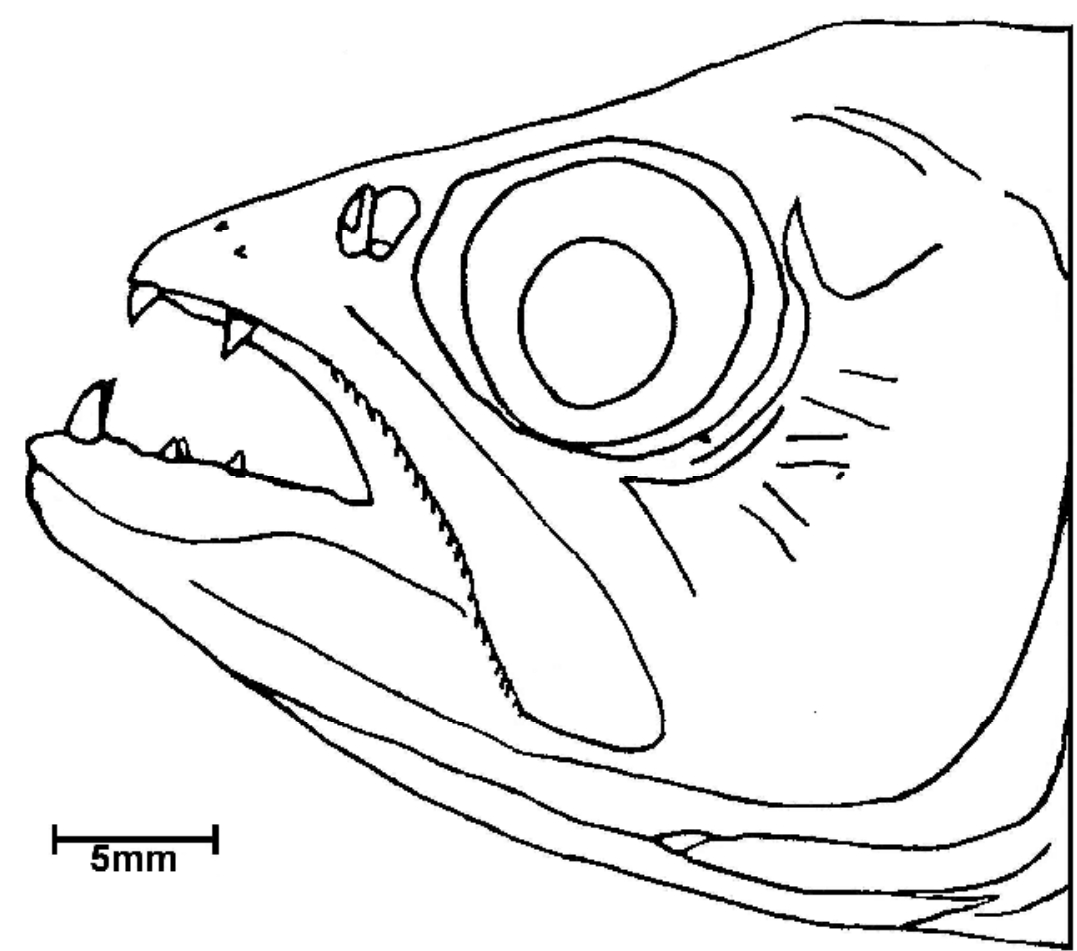

FIGURA 5 - Desenho esquemático da cabeça de Oligosarcus jenynsii com indicação dos dentes.

\section{Análise alimentar}

Dentre os 76 exemplares de 0 . robustus analisados, 35 apresentaram estômagos vazios, destes 12 foram encontrados no verão e 23 no inverno. Para os 88 exemplares de 0 . jenynsii analisados, 32 estavam com os estômagos vazios, sendo oito encontrados no verão e 24 no inverno.
Para as duas espécies constatou-se um maior número de estômagos vazios na estação de inverno.

\section{Dieta de Oligosarcus robustus}

Os itens alimentares mais frequentes na dieta de $O$. robustus foram insetos com frequência de ocorrência (FO) de $58,54 \%$, peixes com $F O=46,34 \%$ e crustáceos com menor frequência $F O=12,2 \%$. No 
entanto, o item peixes foi mais importante em peso (G\%) com 65,6\%, seguido pelo item insetos com
$30,4 \%$ e crustáceos representando apenas $4 \%$ do peso da dieta (Tabela 2).

TABELA 2 - Itens alimentares encontrados na dieta de O. robustus $(n=41)$ e O. jenynsii $(n=56)$; $(F O)$ freqüência de ocorrência e (G\%) percentagem de peso, por grupo taxonômico e por item individual.

\begin{tabular}{|c|c|c|c|c|c|c|c|c|}
\hline & \multicolumn{4}{|c|}{ Oligosarcus robustus } & \multicolumn{4}{|c|}{ Oligosarcus jenynsii } \\
\hline & \multicolumn{4}{|c|}{$(n=41)$} & \multicolumn{4}{|c|}{$(n=56)$} \\
\hline & $\mathrm{FO}$ & G\% & $\underline{\mathrm{FO}}$ & G\% & $\underline{\mathrm{FO}}$ & G\% & $\underline{\mathrm{FO}}$ & G\% \\
\hline & \multicolumn{2}{|c|}{ grupo } & \multicolumn{2}{|c|}{ item } & \multicolumn{2}{|c|}{ grupo } & \multicolumn{2}{|c|}{ item } \\
\hline MOLUSCOS & & & & & 3,57 & 0,10 & & \\
\hline Gastropoda & & & & & & & 3,57 & 0,10 \\
\hline ARACNÍDEOS & & & & & 1,79 & 0,01 & 1,79 & 0,01 \\
\hline CRUSTÁCEOS & 12,2 & 3,99 & & & 35,71 & 3,79 & & \\
\hline Camarão & & & 9,76 & 3,75 & & & 7,14 & 1,20 \\
\hline Aegla sp & & & 2,44 & 0,24 & & & 5,36 & 0,81 \\
\hline Amphipoda & & & & & & & 10,71 & 0,50 \\
\hline Não Identificado & & & & & & & 12,50 & 1,27 \\
\hline INSETOS & 58,54 & 30,4 & & & 83,95 & 79,28 & & \\
\hline Blattodea & & & & & & & 1,79 & 0,54 \\
\hline Coleoptera & & & & & & & 1,79 & 1,10 \\
\hline Diptera & & & & & & & 1,79 & 0,02 \\
\hline Ephemeroptera & & & 36,59 & 29,88 & & & 32,14 & 61,14 \\
\hline Odonata & & & 14,63 & 0,47 & & & 17,86 & 0,25 \\
\hline Orthoptera & & & & & & & 1,79 & 0,02 \\
\hline Plecoptera & & & 2,44 & 0,01 & & & 7,14 & 7,58 \\
\hline Trichoptera & & & & & & & 1,79 & 0,02 \\
\hline Não Identificado & & & 4,88 & 0,06 & & & 17,86 & 8,61 \\
\hline PEIXES & 46,34 & 65,6 & & & 28,57 & 16,82 & & \\
\hline Characidae & & & & & & & 3,57 & 1,41 \\
\hline Gobiidae & & & & & & & 1,79 & 0,81 \\
\hline Oligosarcus jenynsii & & & 2,44 & 6,86 & & & & \\
\hline Hisonotus taimensis & & & 2,44 & 0,38 & & & & \\
\hline Não Identificado & & & 41,46 & 58,36 & & & 23,21 & 14,60 \\
\hline
\end{tabular}


Entre os insetos a maior frequência de ocorrência e importância em peso foi para Ephemeroptera com $\mathrm{FO}=36,6 \%$ e $\mathrm{G}=29,8 \%$ representando $98 \%$ do peso total de insetos na dieta, seguido por Odonata com $\mathrm{FO}=14,6 \%$ e Plecoptera com $\mathrm{FO}=2,4 \%$. O item insetos não-identificados apresentou $\mathrm{FO}=4,9 \%$ (Tabela 2 ).

$O$ item peixes não-identificados registrou grande frequência no grupo com $\mathrm{FO}=41,5 \%$. Entre os peixes identificados foram encontrados Oligosarcus jenynsii (Gunther, 1864) e Hisonotus taimensis (Buckup, 1981) com FO=2,4\% cada (Tabela 2). O grupo dos crustáceos apareceu com pouca frequência de ocorrência, identificando apenas camarão FO=9,8\% e Aegla sp com FO=2,4\% (Tabela 2). Entre os camarões foi identificado um exemplar de Macrobrachium borelli (Nobili, 1896).

No inverno foram analisados 10 estômagos de O. robustus. A preferência neste período foi para o grupo peixes, tendo o item peixes não-identificados registrado frequência de $\mathrm{FO}=70 \%$, com uma alta porcentagem em peso $\mathrm{G}=95,7 \%$ (Fig. 6). Os grupos crustáceos e insetos registraram semelhante frequência de ocorrência $\mathrm{FO}=20 \%$ cada. Entre os crustáceos este valor foi representado pelo camarão e entre os insetos, pela Ordem Ephemeroptera.

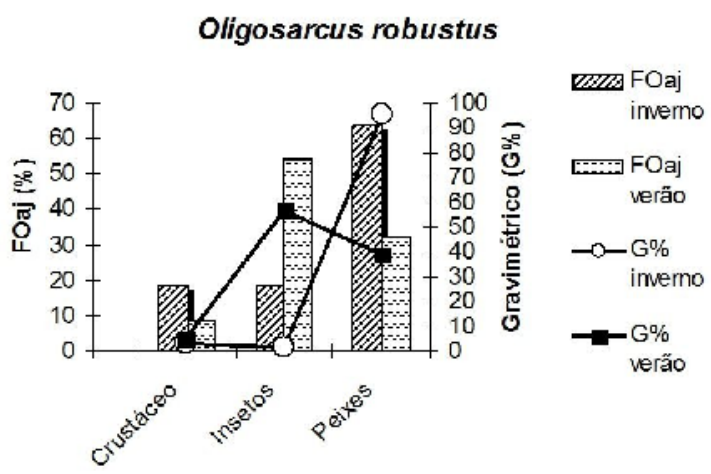

FIGURA 6 - Importância relativa dos diferentes itens alimentares de $O$. robustus, analisados quanto aos métodos de freqüência de ocorrência e gravimétrico, nas estações de inverno e verão; FOaj freqüência de ocorrência ajustada.

No verão foram analisados 31 estômagos de $O$. robustus. A maior diversidade de itens alimentares ocorreu neste período, sendo o item insetos o mais abundante na dieta (Fig. 6). Dentre o grupo dos insetos, Ephemeroptera apareceu com $\mathrm{FO}=41,9 \%$, com elevada importância em peso $\mathrm{G}=54,7 \%$, seguido de Odonata com FO=19,3\% e Plecoptera com
$\mathrm{FO}=3,2 \%$. O item insetos não-identificados totalizou $\mathrm{FO}=6,5 \%$. $O$ item peixes não-identificados somou $\mathrm{FO}=32,3 \%$, com importância em peso de $\mathrm{G}=19,7 \%$.

A frequência de ocorrência $(F O)$ relacionada com a abundância específica da presa (Pi-G\%), (método gráfico de Amundsen) identificou preferência pelo item peixes no inverno e insetos no verão (Figs. 7 e 8).

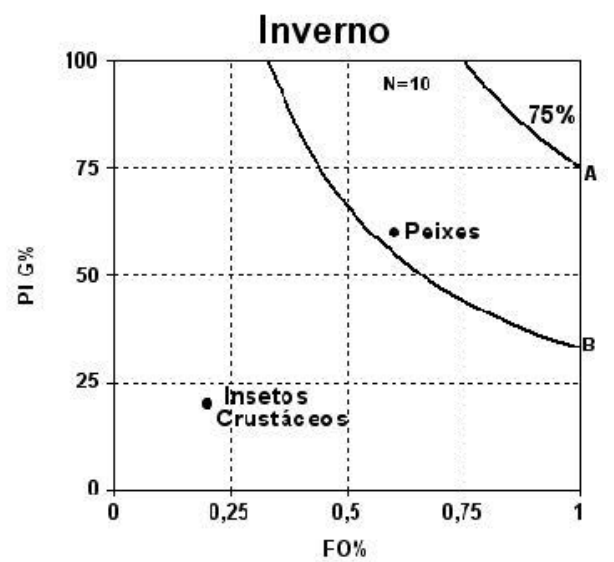

FIGURA 7 - Estratégia alimentar em peso de O. robustus, analisada na estação de inverno (julho/2001); Pi-G\%= abundância específica da presa em peso; $F O=$ Freqüência de ocorrência. As isolinhas $A$ e $B$ representam os níveis de corte. $A=$ nível de corte em $75 \%$; $B=100 / S$, onde $\mathrm{S}=$ número total de presas encontradas nos conteúdos e $\mathrm{N}=$ número de indivíduos analisados. 


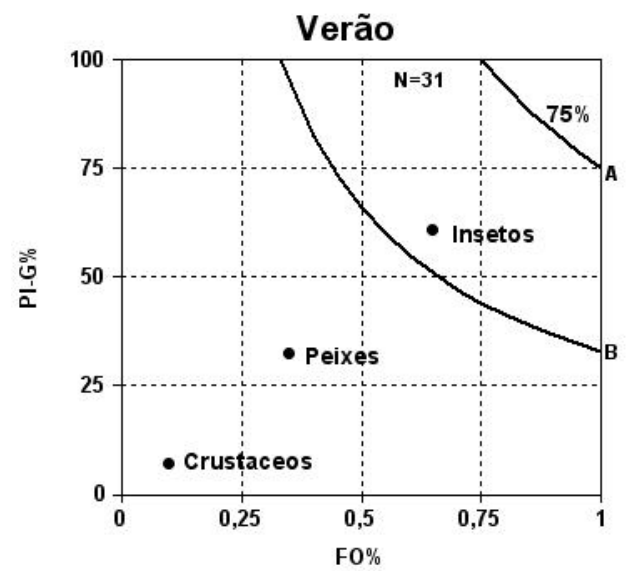

FIGURA 8 - Estratégia alimentar em peso de O. robustus, analisada na estação de verão (dezembro/2001) ; Pi-G\%= abundância específica da presa em peso; $\mathrm{FO}=$ Freqüência de ocorrência. As isolinhas $\mathrm{A}$ e $\mathrm{B}$ representam os níveis de corte. $\mathrm{A}=$ nível de corte em $75 \%$; $\mathrm{B}=100 / \mathrm{S}$, onde $\mathrm{S}=$ número total de presas encontradas nos conteúdos e $\mathrm{N}=$ número de indivíduos analisados.

\section{Dieta de Oligosarcus jenynsii}

Os itens alimentares mais frequentes na dieta de O. jenynsii foram insetos com $\mathrm{FO}=83,9 \%$ e $\mathrm{G}=79 \%$ de importância em peso, crustáceos com $\mathrm{FO}=35,7 \%$ e peso de $3,79 \%$ e peixes com $\mathrm{FO}=28,6 \%$ e $16,8 \%$ em peso (Tabela 3 ).

Tabela 3 - Análise dos itens alimentares de O. robustus e O. jenynsii por estação do ano; (FO) freqüência de ocorrência e (G\%) percentagem de peso.

\begin{tabular}{|c|c|c|c|c|c|c|c|c|}
\hline & \multicolumn{4}{|c|}{ Oligosarcus robustus } & \multicolumn{4}{|c|}{ Oligosarcus jenynsii } \\
\hline & \multicolumn{2}{|l|}{$\begin{array}{c}\text { Inverno } \\
(\mathrm{n}=10)\end{array}$} & \multicolumn{2}{|c|}{$\begin{array}{l}\text { Verão } \\
(n=31)\end{array}$} & \multicolumn{2}{|l|}{$\begin{array}{c}\text { Inverno } \\
(n=20)\end{array}$} & \multicolumn{2}{|c|}{$\begin{array}{l}\text { Verão } \\
(n=36)\end{array}$} \\
\hline & FO & G\% & FO & G\% & FO & G\% & FO & G\% \\
\hline \multicolumn{9}{|l|}{ MOLUSCO } \\
\hline Gastropoda & & & & & 10,00 & 0,22 & & \\
\hline \multicolumn{9}{|l|}{ ARACNIDEO } \\
\hline Aracnideo & & & & & & & 2,78 & 0,01 \\
\hline \multicolumn{9}{|l|}{ CRUSTÁCEO } \\
\hline Camarão & 20,00 & 3,02 & 3,23 & 0,64 & 5,00 & 0,83 & 5,56 & 0,76 \\
\hline Aegla & & & 3,23 & 0,45 & 10,00 & 2,23 & 5,56 & 0,33 \\
\hline Amphipoda & & & & & 15,00 & 1,09 & 8,33 & 0,06 \\
\hline Não identificado & & & & & 10 & 6,14 & 4,55 & 0,01 \\
\hline \multicolumn{9}{|l|}{ INSETOS } \\
\hline Blattodea & & & & & 5,00 & 1,18 & & \\
\hline Coleoptero & & & & & & & 2,78 & 1,91 \\
\hline Diptera & & & & & & & 2,78 & 0,02 \\
\hline Ephemeroptera & 20,00 & 1,27 & 41,94 & 54,79 & 25 & 55,75 & 36,11 & 61,87 \\
\hline Odonata & & & 19,35 & 0,88 & 10 & 0,13 & 22,22 & 0,32 \\
\hline Orthoptera & & & & & & & 2,78 & 0,03 \\
\hline Plecoptera & & & 3,23 & 0,06 & & & 11,11 & 13,12 \\
\hline
\end{tabular}


Continuação

\begin{tabular}{|c|c|c|c|c|c|c|c|c|}
\hline \multirow[b]{3}{*}{ Trichoptera } & \multicolumn{3}{|c|}{ Oligosarcus robustus } & \multicolumn{5}{|c|}{ Oligosarcus jenynsii } \\
\hline & \multirow[t]{2}{*}{ Inverno } & \multirow[t]{2}{*}{ Verão } & \multicolumn{4}{|c|}{ Inverno } & \multicolumn{2}{|c|}{ Verão } \\
\hline & & & & & & & 2,78 & 0,01 \\
\hline Não identificado & & & 6,45 & 0,10 & 25 & 6,12 & 22,22 & 9,58 \\
\hline \multicolumn{9}{|l|}{ PEIXES } \\
\hline Astyanax sp & & & 3,23 & 9,76 & & & & \\
\hline Characidae & & & & & & & 5,56 & 5,66 \\
\hline O. jenynsii & & & 3,23 & 12,85 & & & & \\
\hline Gobidae & & & & & & & 2,78 & 1,81 \\
\hline Hisomotus taimensis & & & 3,23 & 0,71 & & & & \\
\hline Não identificado & 70,00 & 95,71 & 32,26 & 19,77 & 20,00 & 26,32 & 25,00 & 4,54 \\
\hline
\end{tabular}

Entre os insetos a maior ocorrência foi para Ephemeroptera com $\mathrm{FO}=32,1 \%$ e também com maior importância em peso $\mathrm{G}=61,1 \%$, seguido por Odonata com $\mathrm{FO}=17,9 \%$. Foram registrados ainda, os grupos de Plecoptera com FO=7,1\% e Coleoptera, Diptera, Trichoptera, Blattodea e Orthoptera com $\mathrm{FO}=1,8 \%$ cada. $\mathrm{O}$ item insetos não-identificados registrou $\mathrm{FO}=17,9 \% \mathrm{e}$ $\mathrm{G}=8,3 \%$ de importância em peso (Tabela 3).

No grupo dos peixes uma grande porcentagem foi para 0 item peixes não-identificados com $\mathrm{FO}=23,2 \%$ e $\mathrm{G}=14,6 \%$. Entre aqueles identificados, foram encontradas espécies da família Characidae com $\mathrm{FO}=3,6 \%$ e Gobiidae com $\mathrm{FO}=1,8 \%$. No grupo dos crustáceos foram encontrados Amphipoda com $\mathrm{FO}=10,7 \%$, camarão com $\mathrm{FO}=7,1 \%$ e Aegla sp. com
$\mathrm{FO}=5,4 \%$. $\mathrm{O}$ item crustáceos não-identificados apareceu com uma frequência de $\mathrm{FO}=12,5 \%$. Entre os camarões foi identificado um exemplar de Palaemonetes argentinus (Nobili, 1901) e entre os Amphipoda um exemplar de Gammaridea gammarus. Também foi registrado o molusco Gastropoda com $\mathrm{FO}=3,6 \%$ e um aracnídeo, não identificado, com $\mathrm{FO}=1,2 \%$, considerados como um item ocasional na dieta.

No inverno foram analisados 20 estômagos de O. jenynsii. A preferência alimentar da espécie foi pelo grupo insetos (Fig. 9), sendo encontrados Ephemeroptera com $\mathrm{FO}=25 \%$ e alta importância em peso $\mathrm{G}=55,8 \%$ e Odonata com $\mathrm{FO}=10 \%$. O item insetos não-identificados totalizou $\mathrm{FO}=10 \%$.

\section{Oligosarcus jenynsii}

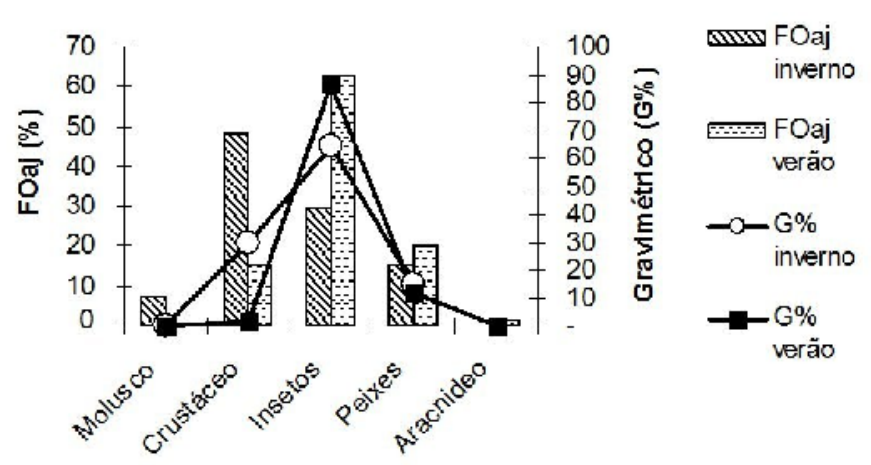

FIGURA 9 - Importância relativa dos diferentes itens alimentares de 0 . jenynsii, analisados quanto aos métodos de freqüência de ocorrência e gravimétrico, nas estações de inverno e verão; FOaj freqüência de ocorrência ajustada.

Entre os crustáceos identificados a maior frequência de ocorrência foi Amphipoda $F O=15 \%$, seguida por camarão $\mathrm{FO}=10 \%$. O item crustáceos não-identificados somou $\mathrm{FO}=25 \%$. $O$ item peixes- 
não-identificados registrou grande importância na dieta com $\mathrm{FO}=20 \%$ e $\mathrm{G}=26,3 \%$.

No verão foram analisados 36 estômagos de $O$. jenynsii. $\mathrm{O}$ item insetos apresentou-se como o mais abundante da dieta (Fig. 9). O grupo Ephemeroptera apresentou elevada frequência $\mathrm{FO}=36,1 \%$ e importância em peso $\mathrm{G}=61,8 \%$, seguido por Odonata (a maioria ninfa) $\mathrm{FO}=22,2 \%$ e Plecoptera $\mathrm{FO}=11,1 \%$ mas com maior importância em peso $(G=13,2)$ que Odonata $(\mathrm{G}=0,32)$. Os grupos Coleoptera, Trichoptera, Diptera, Orthoptera apresentaram $\mathrm{FO}=2,8 \%$ cada um. $\mathrm{O}$ item insetos não-identificados apresentou elevada frequência $\mathrm{FO}=22,2 \%$ e $\mathrm{G}=9,58 \%$, devido ao alto grau de digestão do conteúdo alimentar.
No grupo dos peixes foram encontrados Astyanax alburnus (Hensel, 1870), Cheirodon interruptus (Jenyns, 1842) da família Characidae com $\mathrm{FO}=5,6 \%$, e um da família Gobiidae com $\mathrm{FO}=2,8 \%$. $\mathrm{O}$ item peixes não-identificados registrou elevada frequência $\mathrm{FO}=25 \%$.

O grupo dos crustáceos registrou uma frequência para Amphipoda de $\mathrm{FO}=8,3 \%$, Aegla $s p$ $\mathrm{FO}=5,6 \%$ e camarão com $\mathrm{FO}=5,6 \%$ com baixa importância em peso.

A frequência de ocorrência (FO) relacionada com a abundância específica da presa (Pi-G\%), (método gráfico de Amundsen) identificou a preferência por insetos tanto no inverno, quanto no verão (Figs. 10 e 11).

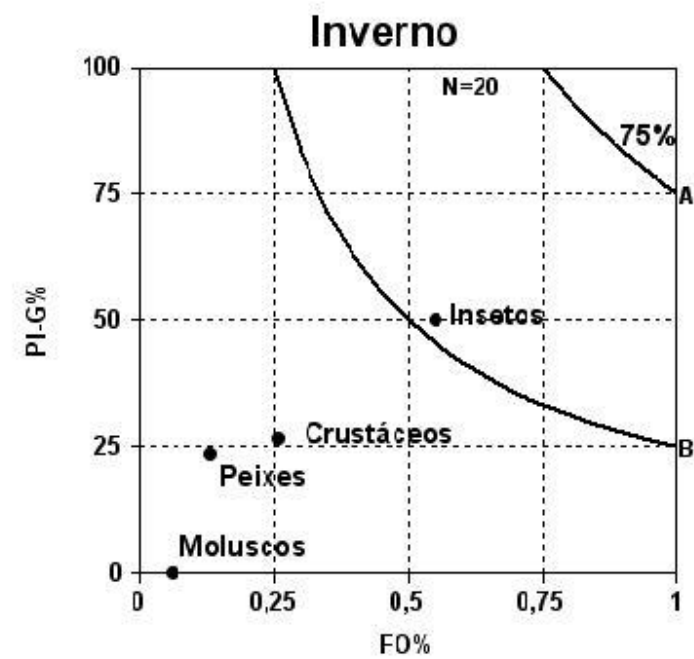

FIGURA 10 - Estratégia alimentar em peso de O. jenynsii, analisada na estação de inverno (julho/2001); Pi-G\%= abundância específica da presa em peso; $F O=$ Freqüência de ocorrência. As isolinhas $A$ e $B$ representam os níveis de corte. $A=$ nível de corte em $75 \%$; $B=100 / S$, onde $\mathrm{S}=$ número total de presas encontradas nos conteúdos e $\mathrm{N}=$ número de indivíduos analisados.

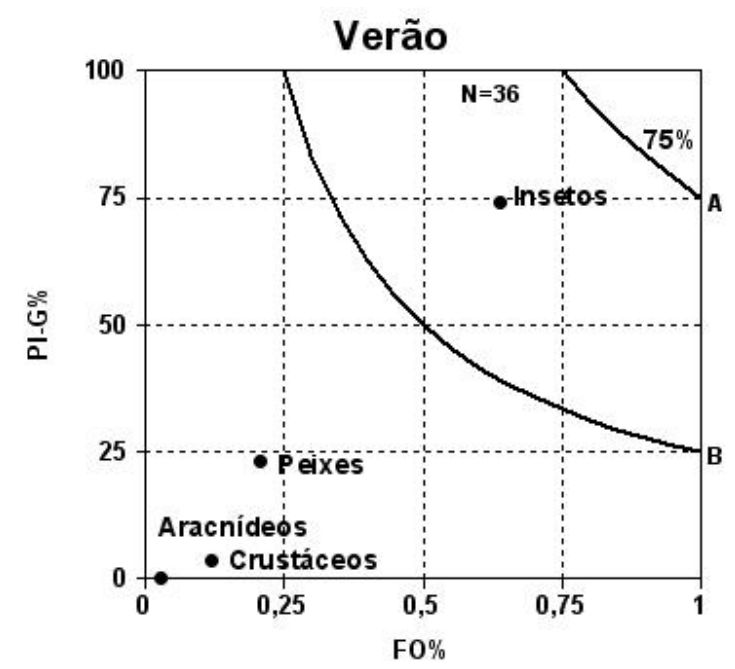

FIGURA 11 - Estratégia alimentar em peso de $O$. jenynsii, analisada na estação de verão (dezembro/2001); Pi-G\%= abundância específica da presa em peso; $F O=$ Freqüência de ocorrência. As isolinhas $A$ e $B$ representam os níveis de corte. $A=$ nível de corte em $75 \%$; $B=100 / S$, onde $\mathrm{S}=$ número total de presas encontradas nos conteúdos e $\mathrm{N}=$ número de indivíduos analisados. 


\section{DISCUSSÃO}

Nas lagoas costeiras do extremo sul do Brasil, duas espécies muito frequentes, Oligosarcus robustus e Oligosarcus jenynsii (Menezes 1969) são caracterizadas, principalmente, pelo número de escamas da linha lateral, pelo comprimento do focinho, bem como pela coloração in vivo da nadadeira caudal que apresenta cor avermelhada em $O$. robustus e amarelada em $O$. jenynsii.

As escamas da linha lateral são pequenas em $O$. robustus, variando entre 75 e 88 , enquanto em $O$. jenynsii as escamas maiores variam entre 51 e 67 . O número de escamas corrobora aquele registrado por Menezes (1969) para $O$. robustus $(75$ a 85) e para $O$. jenynsii (54 a 62), variando pouco em relação aos exemplares analisados.

A necessidade do indivíduo em adaptar-se à captura de diferentes presas, exige uma diferenciação na morfologia das estruturas que compõem a boca. A forma e a posição da boca, a forma e o número de rastros branquiais, são estruturas morfológicas, entre outras, relacionadas à alimentação (Moyle \& Cech 1982, Wootton 1990). A forma da boca em O. robustus é resultado do maior comprimento da maxila sobre a mandíbula, projetando-a para fora. $\mathrm{O}$ focinho torna-se mais proeminente e a boca tem posição terminal inferior, alimentando-se de insetos e peixes da coluna dágua à sua frente. $\mathrm{Em} O$. jenynsii o focinho é mais curto com a mandíbula projetando-se para fora e a boca tem posição superior e seu alimento preferencial são os insetos.

A observação dos rastros branquiais possibilita o conhecimento sobre a dieta do peixe, uma vez que esta estrutura apresenta adaptações diretamente relacionadas ao tamanho do alimento preferencial (Wootton 1990). Os rastros branquiais dos exemplares analisados são extremamente curtos e pouco numerosos (O. robustus $=20-28$ e $O$. jenynsii=19-26), adequados à dieta de grandes partículas, identificados pelos itens mais frequentes que foram insetos, peixes e crustáceos.

No período do inverno a frequencia de insetos nos estômagos das duas espécies foi baixa, menor que no período do verão. Para $O$. jenynsii o grupo insetos foi o mais frequente, enquanto que para $O$. robustus o item mais frequente na dieta alimentar foi peixes, complementada por insetos e crustáceos.
Dentre os insetos somente $o$ item Ephemeroptera foi abundante e expressivo em peso, enquanto Hartz et al. (1996) encontraram uma grande variedade de insetos (Orthoptera, Diptera, Odonata, Hemiptera, Trichoptara, Hymenoptera, Ephemeroptera e Coleoptera) nos estômagos de $O$. jenynsii, na Lagoa Caconde, no norte do estado do RS, fazendo com que insetos seja um dos principais alimentos desta espécie, neste local.

A estratégia alimentar das duas espécies analisadas, no período de inverno, foi generalista com tendência a diferentes itens, sendo que o grupo peixes registrou maior frequência e peso em $O$. robustus durante o inverno.

O verão foi a estação na qual os exemplares apresentaram uma alimentação mais intensa e onde - grupo insetos predominou com elevada diversificação nos estômagos. Entre os insetos o grupo Ephemeroptera foi dominante e com elevado peso, enquanto o grupo Odonata, também dominante, não foi expressivo em peso, por estar a maioria no estágio ninfa. A dominância de Ephemeroptera está relacionada ao maior número de indivíduos capturados na estação de verão, época que coincide com uma grande eclosão destes organismos, que morrem poucas horas após atingirem a fase adulta. Esta eclosão acontece quase sempre ao mesmo tempo, geralmente pouco após o pôr-do-sol, caindo na água logo em seguida, tornando-se uma presa fácil e abundante. A mesma abundância de Ephemeroptera foi relacionada por Moresco \& Bemvenuti (2005), nos estômagos de Trachelyopterus lucenai durante o verão. A preferência alimentar por insetos não foi observada por Hartz et al. (1996), para a dieta de $O$. jenynsii na Lagoa Caconde. Estes autores encontraram peixes como alimento preferencial da espécie.

$O$ item peixes também frequente na estação de verão, para as duas espécies, representou o segundo item no peso do estômago. $O$ item peixes-nãoidentificados registrou elevado peso, devido ao alto grau de digestão do mesmo. Entre os peixes identificados foram encontrados exemplares de Hisonotus taimensis, Cheirodon interruptus, Astyanax alburnus e $O$. jenynsii. Este último foi consumido apenas por $O$. robustus.

O item crustáceo foi pouco frequente no verão, apesar de camarão ser 0 item preferencial para. $O$. 
jenynsii, no Lago São Roque, Argentina (Haro \& Gutiérrez, 1985).

De acordo com Amundsen et al. (1996), a posição do item insetos no canto superior direito do gráfico, abaixo da isolinha dos $75 \%$, indica a estratégia alimentar generalista para as duas espécies. A posição dos itens peixes e crustáceos nestes gráficos, indicam que estes itens foram complementares à dieta das duas espécies.

A inversão do item preferencial na dieta de $O$. robustus entre as estações de inverno (peixes) e verão (insetos), caracteriza um comportamento generalista-oportunista, ou seja, alimenta-se segundo a disponibilidade do alimento mais abundante no ambiente. Para $O$. jenynsii 0 item alimentar preferencial foi o mesmo nas duas estações caracterizando um comportamento generalista com tendência a predar insetos.

A preferência pelo item peixe para $O$. robustus e pelo item inseto para $O$. jenynsii na estação de inverno pode estar relacionado com a competição alimentar entre as duas espécies e sua coexistência no ambiente. Os organismos podem estabelecer uma relação de competição pelo alimento disponível quando ocorrem em um mesmo local e, provavelmente, alimentar-se de diferentes itens, ocupando diferentes hábitats ou ainda utilizando os recursos em diferentes períodos (Hyndes \& Potter 1997). Porém, se o alimento e as estruturas morfológicas utilizadas na alimentação são diferentes entre as espécies, elas podem coexistir e a segregação entre elas não se torna evidente (Wootton 1990). É o que se observa com os dentudos cuja estrutura morfológica da boca tem posição diferente variando a obtenção do alimento entre a coluna dágua e a superfície.

\section{AGRADECIMENTOS}

Aos colegas do Laboratório de Ictiologia da Universidade Federal do Rio Grande, FURG, especialmente ao MSc. Marcelo Dias de Mattos Burns e MSc. Mateus Tavares Kutter pelo apoio nas coletas, auxílio no processamento dos dados e revisões do texto. Ao coordenador do Programa Brasileiro de Pesquisas Ecológicas de Longa Duração (PELD-TAIM) Dr. David da Motta Marques, pelo suporte financeiro nas coletas, aos técnicos de laboratório Nilton A. de Abreu (FURG) e Antonio R. T. Bueno (UFRGS) pelo auxílio nas coletas.

\section{REFERÊNCIAS}

AMUNDSEN, P.A.; GABLER, H.M. \& STALDVIK, F.J. 1996. A new approach to graphical analysis of feeding strategy from stomach contents data- modification of the Costello (1990) method. Norway. Journal of Fish Biology , 48: 607- 614.

BURNS, M.D.M.; GARCIA, A.M.; VIEIRA, J.P.; BEMVENUTI, M.A.; MOTTA-MARQUES, D.L. \& CONDINI, V. 2006. Evidence of habitat fragmentation affecting fish movement between the Patos and Mirim coastal lagoons in southern Brazil. Neotropical Ichthyology 4 (1):69-72.

DELANEY, P.J.V. 1995. Fisiografia e geologia da superfície da Planície Costeira do Rio Grande do Sul. Porto Alegre, UFRGS. v.6:1-195 (Publicação especial da Escola de Geologia).

GARCIA, A.M.; VIEIRA, J.P.; BEMVENUTI, M.A.; MOTTAMARQUES, D.L.; BURNS, M.D.M.; MORESCO, A. \& CONDINI, V. 2006a. Checklist comparison and dominance patterns of the fauna at Taim Wetland, South Brazil. Neotropical Icththyology 4(2):261-268.

GARCIA, A.M; HOINGHAUS, D.J; VIEIRA, J.P.; WINEMILLER, K.O.; MOTTA-MARQUES, D.L.; BEMVENUTI, M.A. 2006b. Preliminary examination of food web structure of Nicola Lake (Taim Hidrological System, south Brazil) using dual $\mathrm{C}$ and $\mathrm{N}$ stable isotope analyses. Neotropical Ichthyology, Porto Alegre, 4 (2): 279-284.

HARO, J.G. \& GUTIÉRREZ, M. 1985. Alimentación de Oligosarcus jenynsii (Günther) (Pisces, Characidae) en el Lago San Roque (Cordoba, Argentina). Rev. Assoc. Cienc. Nat. del Litoral, 16 (2): 227-235

HARTZ, S.M.; MARTINS, A. \& BARBIERI, G. 1996. Dinâmica da alimentação e dieta de Oligosarcus jenynsii (Gunther, 1864) na Lagoa Caconde, Rio Grande do Sul, Brasil (Teleoestei, Characidae). B. Inst. Pesca. São Paulo, 23 (único): 21-29.

HERMES-SILVA, S.; MEURER, S. \& ZANIBONI FILHO, E. 2004. Biologia alimentar e reprodutiva do peixe-cachorro Oligosarcus jenynsii (Gunther, 1864) na região do alto rio Uruguai, Brasil. Maringá 26 (2):175-179.

HYNDES, G.A. \& POTTER, I.C. 1997. Age, growth and reproduction of Sillago schomburgkii in nearshore waters and comparisons of life history strategies of a suite of Sillago species. Envir. Biol. Fish. 49:435-447.

HYSLOP, P.J.1980. Stomach contents analysis - a review of methods and their application. Jour. Fish. Biol. 17: 411- 429.

MENEZES, N.A. 1969. Systematics and evolution of the tribe Acestrorhynchini (Pisces, Characidae). Arquivos de Zoologia, SP. $18(1-2): 150 p$.

MENEZES, N.A. 1987. Três espécies novas de Oligosarcus Gunther, 1864 e redefinição taxonômica das demais espécies do gênero (Osteichthyes, Teleostei, Characidae). Bol. Zool., São Paulo, 11 :1-39.

MORESCO A. \& BEMVENUTI M.A. 2005. Morphologic features and feeding analysis of the black catfish Trachelyopterus lucenai Bertoletti, Pezzi da Silva \& Pereira (Siluriformes, Auchenipteridae). Acta Limnologica Brasiliensia 17(1):37-44.

MOTTA-MARQUES, D.L.; TUCCI, C.; CALAZANS, D.; CALLEGARO, V.L.M. \& VILLANUEVA, A. 2002. Sistema hidrológico do Taim Site 7. IN: SEELIGER, U., CORDAZZO, C.V. \& BARBOSA, F. Os sites e o programa Brasileiro de pesquisas Ecológicas de Longa Duração. Belo Horizonte, 184p. 
MOYLE, P.B. \& CECH, J.J.Jr. 1982. Fishes: An introduction to Ichthyology. Prentice-Hall, Inc. New Jersey 593p.

NUNEZ, D.M. \& HARTZ, S.M. 2006. Feeding dynamics and ecomorphology of Oligosarcus jenynsii (Gunther, 1864) and Oligosarcus robustus (Menezes, 1969) in the Lagoa Fortaleza, southern Brazil. Braz. J. Biol. 66(1A): 121-132.

VILLWOCK, J.A. 1972. Contribuição à geologia do Holoceno da Província Costeira do Rio Grande do Sul. Tese de Mestrado, Instituto de Geociência da UFRGS, Porto Alegre, 113p.
WOOTTON, R.J. 1990. Ecology of Teleost Fishes. New York, Chapman and Hall, 404p.

Submetido $-16 / 05 / 2010$

Aceito $-11 / 10 / 2010$ 